\title{
No reliable evidence that ozone gas stops or reverses tooth decay
}

\author{
Is ozone effective in arresting or reversing the progression of dental caries?
}

\begin{abstract}
Rickard GD, Richardson R, Johnson T, McColl D, Hooper L.
Ozone therapy for the treatment of dental caries (Cochrane Review). In the Cochrane Library. Chichester: John Wiley; 2004, issue 3
\end{abstract}

Data sources The Cochrane Oral Health Group's Trials Register, Cochrane Central Register of Controlled Trials, Medline and Premedline, Embase, CINAHL (Cumulative Index to nursing and Allied Health Literature)! and the Allied and Complementary Medicine Database were searched. The journal Quintessence was searched by hand and KaVo Dental (Biberach, Germany) manufacturers of HealOzone apparatus were contacted for any additional published or unpublished trials.

Study selection Inclusion was assessed independently by at least two reviewers. Trials were only included if they met the following criteria: randomisation in a controlled trial; single surface in vivo carious lesion accessible to ozone application; clear allocation concealment; ozone application to the lesions in the intervention group; no such application of ozone in the control group; and outcomes measured after at least 6 months.

Data extraction and synthesis Reviewers independently extracted information in duplicate. A paucity of comparable data did not allow meta-analytic pooling of the included studies.

Results Three trials were included, with a combined total of 432 randomised lesions (137 participants). Forty-two conference papers, abstracts and posters were excluded (from an unknown number of studies). The risk of bias in all studies appeared high. The analyses of all three studies were conducted at the level of the lesion, which is not independent of the person. For this reason, pooling of data was not appropriate or attempted. Individual studies showed inconsistent effects of ozone on caries, across different measures of caries progression or regression. Few secondary outcomes were reported, but one trial reported an absence of adverse events.

Conclusions Given the high risk of bias in the available studies and lack of consistency between different outcome measures, there is no reliable evidence that application of ozone gas to the surface of decayed teeth stops or reverses the decay process. There is a fundamental need for more evidence of appropriate rigour and quality before the use of ozone can be accepted into mainstream primary dental care or can be considered a viable alternative to current methods for the management and treatment of dental caries.

\section{Commentary}

The cutting edge in cariology is not cavity preparation: current disease-management concepts are better fulfilled by strategies to arrest early caries without destroying the healthy tooth structure. Any recommended clinical treatment requires proof of effect, especially when new technology and novel management concepts are involved. The authors of this Cochrane Review critically evaluated available clinical research concerning ozone treatment

Address for correspondence: Emma Tavender, Review Group Co-ordinator, Cochrane Oral Health Group, Manchester Dental Education Centre, School of Dentistry, University of Manchester, Higher Cambridge Street, Manchester M15 6FH, UK. E-mail: emma.tavender@man.ac.uk and conclude that the evidence is limited. The review reveals a particularly high number of conference abstracts relative to peerreviewed published research.

Ozone gas is a strong oxidising agent with the ability to kill bacteria upon contact. Since carious lesions must be accessible, the research largely involves root caries and occlusal fissures. Clinical evidence of significant initial microbicidal effect has recently been published for root caries. ${ }^{1}$ The lack of control lesions and of any monitoring of bacterial recolonisation compromise the value of the 3.0-5.5-month follow-up, however.

There is no evidence that ozone-treated oral lesions do not become re-infected over time. A private-practice controlled clinical trial using multiple (five) applications over 18 months has noted significant root lesion hardening. ${ }^{2}$ Only 'leathery' root lesions were treated in these studies but it has been shown that aggressive conventional preventive treatment can be effective in controlling this type of root caries. ${ }^{3}$

Fissures are one of the most caries-prone plaque stagnation sites. Preventive strategies other than sealants have proved relatively ineffective to date. Ten-year radiological evidence indicates that sealed restorations can halt the progress of even established dentinal caries. ${ }^{4}$ Important questions, therefore concerning ozone treatment including depth, consistency, duration and significance of microbicidal effect remain. The treatment frequency and longterm outcomes on different types of lesions and populations are still uncertain.

Practitioners need to be aware of the limitations of the available data. This Cochrane Review should be of immense value to stimulate and guide appropriately conducted clinical validation studies. Until then the jury is still out on the clinical implications of this emerging technology.

\section{Practice point}

- There is a need for more evidence of appropriate rigour before the clinical implications of this emerging technology can be evaluated.

\section{Dorothy McComb}

Director of Comprehensive Care Programme, Faculty of Dentistry, University of Toronto, Toronto, Ontario, Canada

1. Baysan A, Lynch E. Effect of ozone on the oral microbiota and clinical severity of primary root caries. Am J Dent 2004; 17:56-60.

2. Holmes J. Clinical reversal of root caries using ozone, double-blind, randomised, controlled 18-month trial. Gerodontology 2003; 20:106-114.

3. Billings RJ, Brown LR, Kaster AG. Contemporary treatment strategies for root surface dental caries. Gerodontics 1985; 1:20-27.

4. Mertz-Fairhurst EJ, Curtis Jr JW, Ergle JW, Rueggeberg FA, Adair SM. Ultraconservative and cariostatic sealed restorations: results at year 10. J Am Dent Assoc 1998; 129:55-66.

Evidence-Based Dentistry (2005) 6, 34.

doi:10.1038/sj.ebd.6400322 